\title{
Femtocell versus WiFi - A Survey and Comparison of Architecture and Performance
}

\author{
Syed Faraz Hasan, Nazmul Haq Siddique and Shyam Chakraborty \\ Intelligent Systems Research Centre \\ Ulster University \\ farazhasan-s@email.ulster.ac.uk,_nh.siddique,ss.chakraborty\}@ulster.ac.uk
}

\begin{abstract}
Femtocells use common cellular air access technologies, but claim to improve system capacity according to Shannon's law by reducing distance between transmitter and receiver and thus improving the signal-to-noise ratio (SNR). Femtocells, however, use the IP Network as backhaul architecture instead of the conventional cellular network infrastructure. Thus, femtocell and WiFi infrastructure networks have a lot in common. This raises a curiosity whether femtocell technology would replace existing WiFi technology. This can be answered only by carefully analyzing the similarities and differences between the two technologies. This paper provides a technical comparison between femtocells and WiFi in terms of architecture, operation and standards.
\end{abstract}

Keywords: AIPN, Backhaul, Convergence, Femtocells, WiFi

\section{Introduction}

Advantages of getting wireless have introduced many technologies into market, primarily through two different directions. The first direction is the cellular systems, which cater primarily for voice services with mobility. However, cellular systems are also increasingly used for various data services. On the other hand, the Wireless Local Area Networks (WLANs) were developed as an extension of the terrestrial LANs, for providing network connectivity with restricted mobility. While cellular networks consist of a dedicated terrestrial backbone, WLANs, on the other hand, commonly connect directly to the IP networks through Digital Subscriber Loop (DSL) or Ethernet backbone network.

Femtocell is a recent technology which uses the IP backbone network along with small-size base stations, based on cellular technology, located indoors. Doing so, femtocells support compatibility with the cellular systems, and at the same time, provide better indoor signal strength [1], commonly unattainable by macro- cell coverage operating at higher frequencies. Interestingly, with the introduction of the femtocell technology, the cellular systems come closer to WiFi through architecture, operating frequency, services offered and data rates. However, there are issues that distinguish them and would lead to acceptance of one technology over the other. This article addresses a preliminary comparison of femtocell technology with WiFi networks to understand dynamics of the market in future.

The rest of the article is organized as follows. Section 2 gives a brief overview of femtocells and WiFi. Benefits of using femtocells in terms of signal loss are highlighted in section 3. Section 4 discusses Network Convergence. Section 5 deals with the technical differences between femtocells and WiFi. This article is concluded at Section 6.

\section{Femtocells and WiFi}

Improving signal strength indoors has been a challenge for all cellular service providers. When radio waves penetrate through the walls, they face attenuation. Consequently, the Received Signal Strength (RSS) at an indoor site is reduced. This attenuation is more prominent at higher frequency bands that are increasingly used for higher bit rate operations. The femtocell base stations (commonly known as Femtocell Access Points or FAP) have emerged as indoor radio base stations (BS) enabling users of a certain cellular service (UMTS, CDMA2000 etc) and WiMAX to make all indoor calls over their internet broadband connection, thus eliminating reduced RSS problem inside buildings [2].

\section{Table 1: Femtocell and WiFi Specifications}

\begin{tabular}{|c|c|c|}
\hline & Femtocell & WiFi \\
\hline Data rates & $7.2-14.4 \mathrm{Mbps}$ & 11 and $54 \mathrm{Mbps}$ \\
\hline Op. Frequency & $1.9-2.6 \mathrm{GHz}$ & 2.4 and $5 \mathrm{GHz}$ \\
\hline Power & $10,100 \mathrm{~mW}$ & $100,200 \mathrm{~mW}$ \\
\hline Range & $20-30 \mathrm{~m}$ & $100-200 \mathrm{~m}$ \\
\hline Services & $\begin{array}{c}\text { Primarily Voice, } \\
\text { and data }\end{array}$ & $\begin{array}{c}\text { Primarily Data, } \\
\text { and voice }\end{array}$ \\
\hline
\end{tabular}




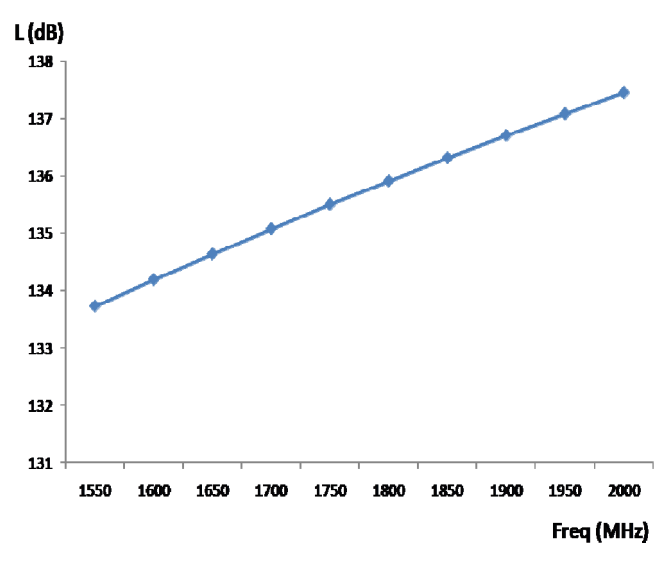

Figure 1: Observations of $\mathrm{OH}$ Model for predicting path loss at different frequencies

Being a direct successor of the cellular systems, femtocells primarily cater for voice services, but data services are also supported in the similar lines of cellular systems. Femtocells are designed to operate on low transmit power $(100 \mathrm{~mW}$ or even $10 \mathrm{~mW})$ in order to avoid interference with similar nearby devices. The femtocell technology offer data rates from 7.2 $14.4 \mathrm{Mbps}$, and with the advances in LTE/SAE, it will increase to $100 \mathrm{Mbps}$.

WLANs, on the other hand, are direct successors of terrestrial local area networks (LAN), for catering data services. In the infrastructure mode of operation, network connectivity is provided in the coverage areas (foot prints) of the Access Points (APs). Besides data services, WLANs are becoming popular for provisioning of VoIP services too. The WLAN specifications are standardized through the IEEE $802.11 \mathrm{a} / \mathrm{b} / \mathrm{g}$ standards. The three versions operate at $2.4 \mathrm{GHz}(802.11 \mathrm{~b} / \mathrm{g})$ and $5 \mathrm{GHz}(802.11 \mathrm{a})$ frequency bands with data rates ranging from $6-54 \mathrm{Mbps}$. However in actual practice, 802.11g WLAN achieves about 30Mbps [3]. Table 1 provides a basic comparison of WLAN and femtocell parameters.

\section{Path Loss Observations}

Okumura Hata (COST-231) Model (1) is very commonly used in predicting path losses and field strengths in the $1500-2000 \mathrm{MHz}$ band in a macro-cell environment over a range of $1-100 \mathrm{~km}$ for different heights of cellular towers [4].

$$
\mathrm{L}_{\mathrm{dB}}=\mathrm{F}+\mathrm{B} \log _{10}(\mathrm{R})-\mathrm{E}+\mathrm{G}
$$

Where,

$\mathrm{F}=46.3+33.9 \log _{10} \mathrm{f}_{\mathrm{c}}-13.82 \log _{10} \mathrm{~h}_{\mathrm{b}}$,

$\mathrm{B}=44.9-6.55 \log _{10} \mathrm{~h}_{\mathrm{b}}$,

$E=\left(1.1 \log _{10} f_{c}-0.7\right) h_{m}-\left(1.56 \log _{10} f_{c}-0.8\right)$ and

$\mathrm{G}=3 \mathrm{~dB}$ for metropolitan areas.
We evaluate path loss L using $\mathrm{OH}$ model, assuming $35 \mathrm{~m}$ base station tower height $\left(\mathrm{h}_{\mathrm{b}}=35 \mathrm{~m}\right), 1.6 \mathrm{~m}$ receive antenna height $\left(\mathrm{h}_{\mathrm{m}}=1.6 \mathrm{~m}\right), 1 \mathrm{~km}$ separation between tower and mobile $(\mathrm{R}=1 \mathrm{~km})$ at different frequencies $\left(\mathrm{f}_{\mathrm{c}}\right)$ ranging from 1500 to $2000 \mathrm{MHz}$. Figure 1 show that path loss increases with increasing carrier frequency, as mentioned in section 2. On the other hand, indoor propagation is commonly modelled using modified Keenan Motley equation (2) [5]. Indoor losses using KM Model are evaluated at different transmitter receiver distances for a typical two room set up.

$\mathrm{L}_{\mathrm{dB}}=32.5+20 \log _{10}(\mathrm{f})+20 \log _{10}(\mathrm{~d})+\left(\mathrm{N}_{\mathrm{W}} \mathrm{XW}\right)$

Where $\mathrm{f}$ is the carrier frequency, $\mathrm{d}$ is transmitterreceiver separation; $\mathrm{N}_{\mathrm{w}}$ is the number of walls and $\mathrm{W}$ is the wall loss factor. Observed values of path loss $\mathrm{L}$ at $\mathrm{f}=1800 \mathrm{MHz}, \mathrm{N}_{\mathrm{w}}=1$ and $\mathrm{W}=2$ and $5 \mathrm{~dB}$ for plasterboard and reinforced concrete walls respectively, over different transmitter-receiver separations are shown in figure 2. Figure 3 depicts a typical set up where transmitter and receiver are placed in adjacent rooms $\left(11 \times 13 \mathrm{~m}^{2}\right.$ dimensions $)$ with plasterboard or reinforced concrete walls. It can be inferred from figures 1 and 2 that femtocells result in reduced power loss when compared with cellular systems.

\section{Network Convergence}

Network convergence refers to using a single interface and a single backbone network for a variety of telecommunication services, for example, voice, video and data. Provisioning of multiple services by means of network convergence has shown benefits for businesses in terms of cost and user productivity [6].

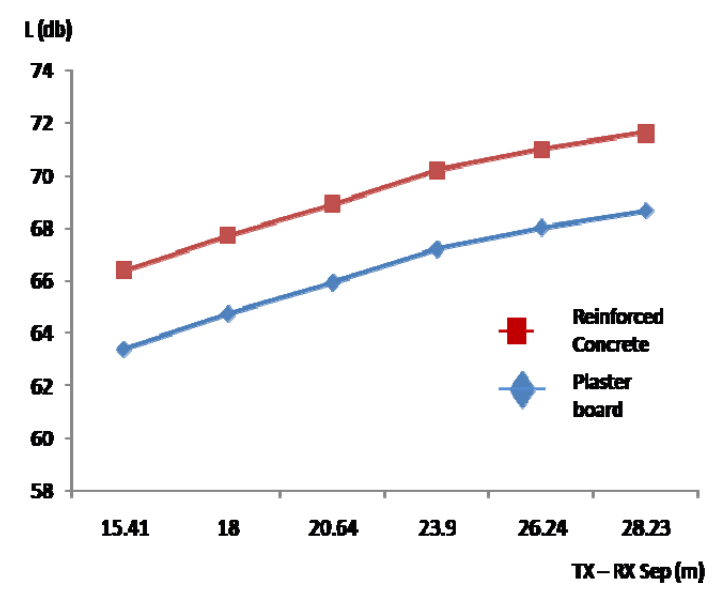

Figure 2: Observations of KM Model for predicting indoor path loss 


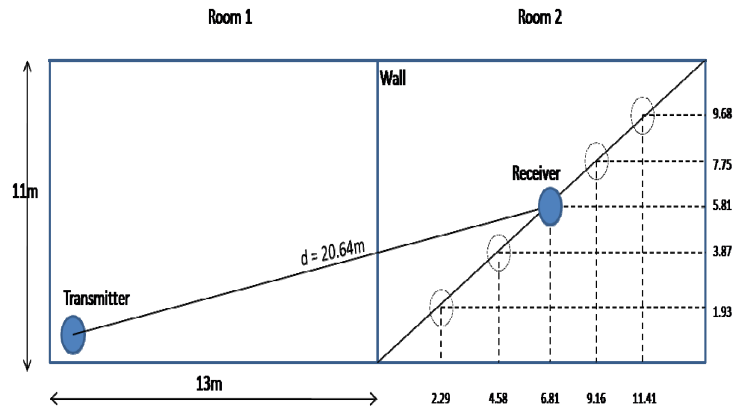

Figure 3: Plan of two adjacent rooms with femtocells

The concept of network convergence evolved through circuit switched Integrated Services Digital Network (ISDN), and gradually migrated to Packet Switched networks, firstly, the Asynchronous Transfer Mode (ATM) based broadband ISDN, and ultimately the IP based Internet. The main objective of advancing in network convergence is to achieve IP convergence in the form of All IP Networks (AIPNs). AIPNs not only provide data, video and voice services over a single IP network but also facilitate connectivity to other external networks [9]. In this way, users can achieve ubiquitous connectivity independent of devices and location.

The Fixed-Mobile Convergence (FMC) is a popular trend in the market, which allows seamless connectivity between fixed and mobile telephone networks. Service providers can bring all potential wireless and wired users under their realm over such integrated environment [7]. This also provides users with the benefits of ubiquitous connectivity. Using the same concept, OMA (Open Mobile Alliance) is converging WPAN (Wireless Personal Area Networks) and Cellular Networks as Converged Personal Network Services (CPNS) [8]. Basic infrastructure of CPNS is proposed to connect several WPAN devices to a mobile phone, which in turn is connected to the cellular system. In this sense, a mobile phone acts as a gateway between WPAN and Cellular Network.

\subsection{Cellular - Femtocell and Cellular - WiFi Convergence}

Technically a Femtocell is a low power cellular base station designed for indoor use typically in Small Office- Home Office (SOHO) environment to provide voice and broadband services. Three different connections of femtocells with circuit switched, packet switched and IMS are shown in Figure 4. In one of them, the Femtocell Access Point FAP is connected to the cellular network via the IP network through a Femtocell Gateway (FGW). FGW routes all indoor calls over the IP network instead of cellular network, thus enabling a closer proximity of the FAPs to mobile nodes. It not only increases system capacity by reducing the load on base station but also allows signals to reach end users without having to penetrate through urban structures like buildings. Notice that, the same air interface can be used both at the femtocell and macro-cell levels.

Cellular - WiFi Convergence on the other hand, refers to simultaneous provisioning of cellular services (e.g. GSM, UMTS, and CDMA etc) and WiFi services (IEEE $802.11 \mathrm{a} / \mathrm{b} / \mathrm{g}$ ) via a single integrated network. A typical example of such an environment is Unlicensed Mobile Access (UMA), in which a dual mode handset makes calls through the internet when WiFi signals are available and through the cellular network otherwise [10]. In this way, access is provided through licensed and unlicensed networks simultaneously, with the use of the UMA Network Controller (UNC), as in figure 5. In order to reap convergence benefits, FGW and UNC both must be light weight, cost effective, scalable and adaptable to changing protocols.

\section{Technical Differences}

WiFi and Femtocells both face similar issues relating to access methods. Rights of accessing a network may be public (open to all subscribers) or private (open to specific subscribers). Public access method provides better QoS and throughput but at the same time increases the number of hand overs and hence adds to the signalling overhead. Private access method is shown to decrease system throughput by $15 \%$, however, surveys suggest that end users prefer using Private Access Methods [11]. Access methods for Femtocells and WiFi networks differ in terms of security. While femtocells allow same security protocols as cellular service, WiFi networks specify their own protocols such as Wired Equivalent Protocol (WEP) or Universal Access Method (UAM) as proposed for Drive-Thru Network Architecture [12].

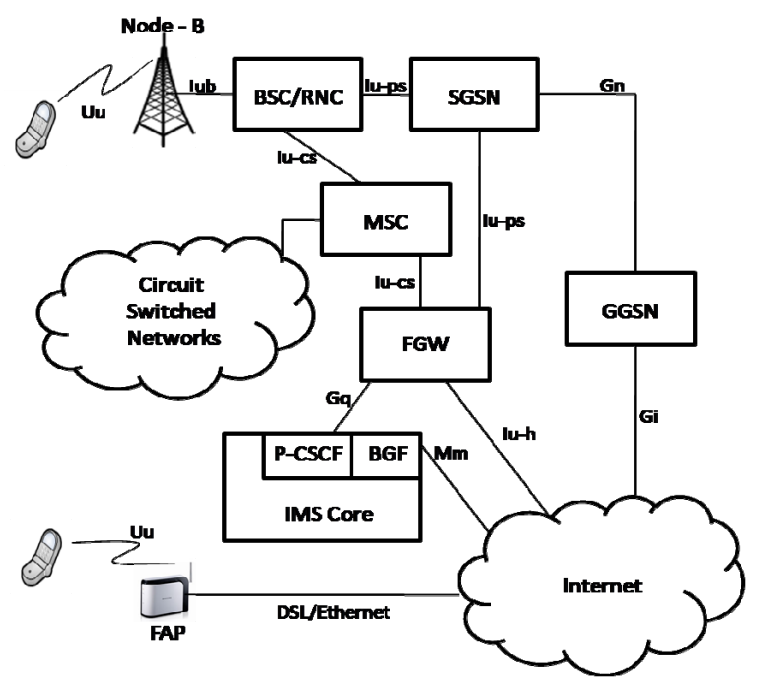

Figure 4: Femtocell Network Architecture 


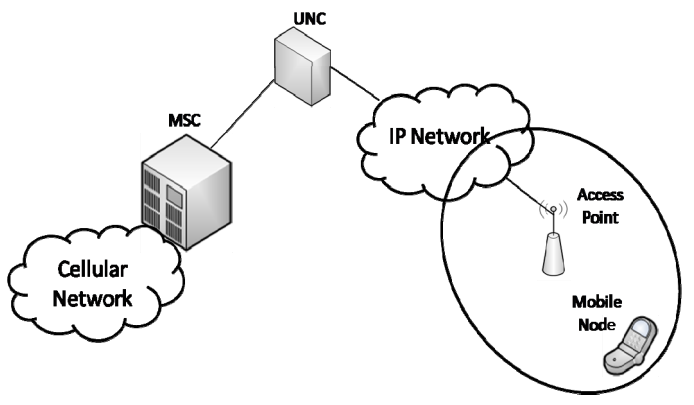

Figure 5: Cellular/WLAN convergence architecture

\subsection{QoS Challenges}

Quality of Service is the ability of packet switched network to provide different priorities to different applications in order to guarantee a certain level of performance for data flow. The 3GPP QoS architecture [13] classifies data into conversational, streaming, interactive and background traffic classes, each having separate QoS attributes. WiFi QoS mechanisms are composed of Service Differentiation (assigning different parametric values of channel accesses to different traffic classes), Admission Control \& Bandwidth Reservation (allowing channel access based on channel measurements and estimations) and Rate Adaptation (changing data rates according to variations in channel conditions). Recently, interoperability between IEEE 802.11 and DiffServ (Differentiated Services) is being explored. Femtocells, on the other hand, might face several hardware changes before providing adequate QoS requirements to users. Using a traffic classifying service, such as DiffServ, may be one method of providing QoS in femtocells. However, it would mean additional bits (DSCP) are encoded every time a retransmission occurs. A leading communication services provider, Oyster, propose $\mathrm{Iu}+$ interface (after making changes in Iu interface transport layer) between femtocells and access controllers to offer QoS over IP network.

\subsection{Frequency Bands}

Femtocell shares the licensed electromagnetic spectrum allocated to cellular service providers. Here two different approaches can be used. One is the Cochannel Frequency Deployment in which, femtocell and macro cell use the same frequency band. An obvious problem with this approach is co-channel interference. However, this problem can be reduced by using for example, Dynamic Frequency Planning [14] for WiMAX femtocells. Orthogonal Channel Deployment is another approach in which, macrocells and femtocells use separate channels. This, on one hand, results in smaller co-channel interference, but on the other hand, may result in reduced overall system capacity. However, since WiFi networks use different
ISM frequency band, that are independent of any particular cellular service, they have no deployment issues. WiFi uses unlicensed ISM band which is available for public use. It poses a problem of interference when too many devices located closed to a WiFi network use the same band.

\subsection{Handsets}

Ordinary cell phones do not allow calls over IP networks. Most WiFi users either use ordinary phones along with a special adapter or Personal Computers to make VoIP calls. Another way is to use special purpose dual-mode which enables phone calls both over IP and cellular networks. In femtocells, an ordinary cellular phone can use the resources of both cellular and IP networks. This is because a femtocell controller provides the interface between IP and cellular networks.

\subsection{Interference}

WiFi faces interference with all devices working on the same unlicensed band in close vicinity. A minimum distance of about $5 \mathrm{~m}$ is recommended to avoid such interferences [15]. Since femtocells operate on same band as macrocells, femtocell devices may face adjacent channel interference with the devices being served by the macro cell. Two femtocells of the same service provider located close to each other might also cause interference. This can be seen as a practical situation where two femtocells of same service provider serve users on two adjacent floors of a building. A study in [16], however, shows that such interferences are not significant because structural obstacles do not let low-powered FAP signals to penetrate through them.

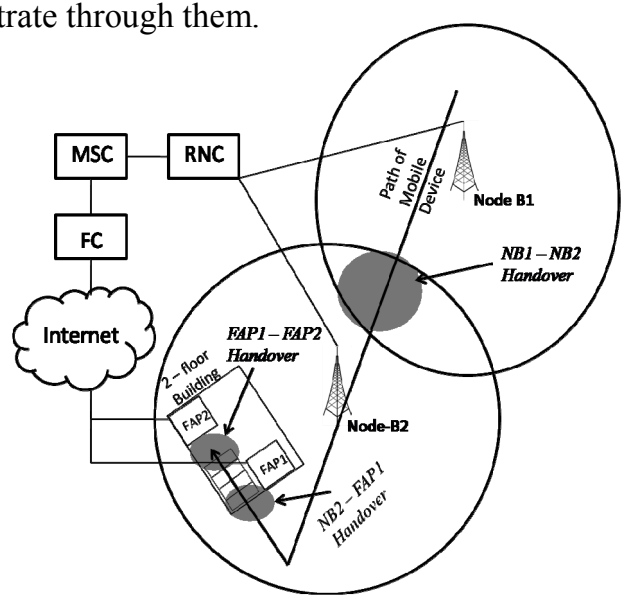

Figure 6: Handovers in femtocell deployed cellular network

\subsection{Hand over}

Mobiles in WiFi networks hand over from one access point to another when the RSS of former becomes lower than a certain threshold. Hand-overs occur between different Access Points randomly placed at 
different geographical locations. In a Cellular/WLAN convergence scenario, shown in figure 5, handovers can also occur between a cellular BS and WiFi AP. In this convergent network, a dual-mode handset will undergo handover from a WiFi AP to a Cellular BS as soon as it gets out of the AP footprint and vice versa. In case of cellular networks and femtocells, mobiles face at least three kinds of hand-overs. One is the normal hand-over, occurring when user moves from one base station to another. This is a BS-BS handover that occurs between two BS-s. This kind of hand-over also happens in infrastructure $\mathrm{WiFi}$ as inter $\mathrm{AP}$ handover. Another kind of handover occurs between cellular BS-s and FAP when mobile moves from outdoors to indoors or vice versa. When a mobile is outdoors, its requests are responded by the cellular base station. As soon as the mobile moves indoors, it starts being served by the femtocell access point instead of cellular BS. This hand-over requires a certain level of synchronization between FAP and Cellular BS because there is no central coordination between the two. A third kind of hand-over scenario is when a user moves from one floor of a building (served by one FAP) to another (served by another FAP associated with same cellular service), that is, a FAP - FAP hand over. These hand overs are shown in figure 6 .

\section{Conclusion}

This paper outlines different issues associated with two competing technologies Femtocells and WiFi. Femtocells and WiFi both have the potential of providing convergent communications services, however, femtocells are reserved for indoor use only. Both use IP network as backhaul, therefore, their performance is affected by IP network conditions. Evaluating their performances under the varied conditions of IP networks is an interesting area of future work. An important issue in this femtocell-WiFi comparison is cost, which is not considered here.

\section{References}

[1] V Chandrasekhar and J Andrews, "Femtocell Networks: A Survey", IEEE Communications Magazine, Vol. 46, no. 9, pp 59-67, 2008.

[2] R Tang, "Indoor Propagation in Cellular/PCS System Design", Wireless Communications and Systems, IEEE 1999 Emerging Technologies Symposium, pp 8.1 - 8.4, 1999.

[3] J Reynolds, "Going Wi-Fi: A Practical Guide to Planning and Building an 802.11 Network", Focal Press, 2003.
[4] S Ranvier, "Path Loss Models", S-72.333 Lectures Physical Layer Methods in Wireless Communication Systems, Radio Laboratory, HUT, 2004.

[5] Ericsson Radio Systems, "Cell Planning for GSM Indoor Systems”, Section 8.3.2, 1998.

www.lautech.edu.ng/eResouces/Indoor\%20Guidelines. pdf (As on April 08, 2009).

[6] Cisco Systems, "How does Network Convergence support a business strategy"

http://www.cisco.com/global/EMEA/sitewide_assets/p dfs/tdm/iptel/roi benefits paper.pdf (As on April 08, 2009).

[7] "Beyond the Quadruple Play: Networking, Convergence and Customer Delivery", edited and published by International Engineering Consortium, 2007.

[8] Work Item WID - 179, Open Mobile Alliance (OMA), Vol. 3, 2008.

http://www.openmobilealliance.org/News/Quarterly/V olume3.aspx\#tf (As on April 08, 2009).

[9] F Ivanek, "Convergence and Competition on the way toward 4G: Where are we going?", IEEE Radio and Wireless Symposium, pp. 256 - 258, 2007.

[10] N Seel, "Business Strategies for the Next Generation Networks", CRC Press 2006.

[11] D Lopez-Perez, A Valcarce, G Roche, E Liu and J Zhang, "Access Methods to WiMAX Femtocells: A downlink system-level case study", IEEE ICCS 2008, pp 1657 - 1662, 2008.

[12] J Ott and D Kutscher, "Exploiting Regular HotSpots for Drive-thru Internet", Proceedings of KiVS 2005.

[13] S Chakraborty, $\mathrm{T}$ Frankkila, J Peisa, P Synnergren, "IMS Multimedia Telephony over Cellular Systems", Wiley, 2007.

[14] D Lopez-Perez, G Roche, A Valcarce, A Juttner and J Zhang, "Interference Avoidance and Dynamic Frequency Planning for WiMAX Femtocell Networks", IEEE ICCS, pp 1579 - 1584, 2008.

[15] N Kushiro, "Performance of Ad Hoc wireless Network on $2.4 \mathrm{GHz}$ Band in Real fields", IEEE transactions on Consumer Electronics, Vol. 54, pp. 80 -86 , No. 1, 2008.

[16] H Claussen, "Performance of Macro and CoChannel femtocells in a hierarchical cell structure", International Journal of Wireless Information Networks Vol. 15, No. 3, pp. 137-147, 2008. 